\title{
Ressources pour lutter contre la stigmatisation liée à la sexualité, à la toxicomanie et aux infections transmissibles sexuellement et par le
}

\section{sang}

\author{
R MacLean ${ }^{1 *}$
}

\section{Résumé}

Contexte : La stigmatisation est largement reconnue comme un obstacle important à la prévention, à la prise en charge et au traitement des infections transmissibles sexuellement et par le sang (ITSS) au Canada. Malgré les avancées majeures en matière de prévention et de traitement des ITSS et les efforts mondiaux pour réduire la stigmatisation, les personnes vivant avec une ITSS continuent de se faire stigmatiser dans les milieux de la santé et des services sociaux au Canada.

Objectif : Décrire l'élaboration, le contenu et l'évaluation des ressources d'application des connaissances et des ateliers de formation conçus pour fournir aux professionnels de la santé et des services sociaux les connaissances et les compétences nécessaires afin d'offrir des services de santé sexuelle, de réduction des effets nocifs et en matière d'ITSS qui soient plus respectueux et plus inclusifs.

Méthodologie : Après avoir effectué une analyse documentaire, une analyse du contexte et des entrevues avec des répondants clés, l'Association canadienne de santé publique (ACSP) a produit un cadre conceptuel, puis a élaboré, en partenariat avec divers organismes et experts communautaires, quatre ressources d'application des connaissances et trois ateliers de formation. Les ressources ont été rédigées et révisées par des fournisseurs de services aussi bien que par des personnes touchées par des ITSS. Les ateliers ont été conçus, mis à l'essai, puis évalués à l'aide de questionnaires remis après les ateliers.

Résultats : Les quatre ressources élaborées étaient les suivantes : un outil d'autoévaluation lié aux ITSS et à la stigmatisation; un guide de discussion à l'intention des fournisseurs de services visant à favoriser des discussions respectueuses et inclusives sur les questions liées à la sexualité, à la toxicomanie et aux ITSS; une trousse d'outils axée sur la réduction de la stigmatisation, la protection de la vie privée, la confidentialité et la criminalisation de la non-divulgation de la séropositivité; ainsi qu'un outil d'évaluation organisationnelle relatif aux ITSS et à la stigmatisation à l'intention des milieux de la santé et des services sociaux. Ces ressources d'application des connaissances ont ensuite été intégrées au contenu de trois formations en personne qui ont été mises à l'essai et évaluées à travers le pays. Le taux de réponse global à l'évaluation après l'atelier a été de $85 \%$; 88 \% des participants ont signalé une meilleure connaissance des diverses formes de stigmatisation, $87 \%$ ont noté une plus grande aisance à discuter de sexualité, de toxicomanie et de réduction des effets nocifs avec leurs clients ou patients, $90 \%$ ont affirmé mieux connaître les stratégies organisationnelles permettant de réduire la stigmatisation et $93 \%$ ont déclaré être en mesure d'intégrer les apprentissages de l'atelier dans la pratique. On a aussi relevé un appui très solide au perfectionnement professionnel sur les enjeux touchant la réduction de la stigmatisation liée aux ITSS.

Conclusion : Ces ressources d'application des connaissances et ces ateliers de formation forment un ensemble complet d'outils élaborés au Canada, que les fournisseurs de services peuvent utiliser pour aider à réduire la stigmatisation lorsqu'ils soignent des clients ou patients atteints d'une ITSS ou d'un trouble connexe. L'évaluation révèle que les fournisseurs de services sociaux et de santé sont très disposés à participer à des occasions d'apprentissage dans ce domaine et que la participation aux ateliers de formation a mené à une sensibilisation accrue et à une volonté d'adopter de meilleures pratiques.

\section{Afilliation}

${ }^{1}$ Association canadienne de santé publique, Ottawa (Ontario)

*Correspondance: rmaclean@ cpha.ca
Citation proposée : MacLean R. Ressources pour lutter contre la stigmatisation liée à la sexualité, à la toxicomanie et aux infections transmissibles sexuellement et par le sang. Relevé des maladies transmissibles au Canada. 2018;44(2):69-75. https://doi.org/10.14745/ccdr.v44i02a05f 


\section{Introduction}

La stigmatisation, définie comme un processus dynamique de dépréciation qui discrédite considérablement un individu aux yeux des autres, est largement reconnue comme un obstacle à la prévention, à la prise en charge et au traitement des infections transmissibles sexuellement et par le sang (ITSS), comme le $\mathrm{VIH}$, les hépatites $\mathrm{B}$ et $\mathrm{C}$, le virus du papillome humain, I'herpès génital et la syphilis (1). Chez les personnes ayant contracté une ITSS, la stigmatisation peut mener à un faible niveau de santé et de bien-être, notamment à des problèmes de santé mentale, au retrait social, à la crainte de déclarer l'ITSS et à une diminution du bien-être sexuel (2-5). Malgré les avancées majeures en matière de prévention et de traitement des ITSS et les efforts mondiaux pour réduire la stigmatisation, de nombreuses études canadiennes ont cerné les milieux de la santé et des services sociaux comme des sources importantes de stigmatisation pour les personnes touchées par une ITSS, et notamment pour celles qui sont infectées par le VIH (3,6-8). La stigmatisation vécue dans les milieux de la santé et des services sociaux peut affecter l'accès et le recours aux services de prévention, de traitement et de soutien ainsi que l'adoption de comportements préventifs relativement aux ITSS, notamment I'observance du traitement antirétroviral pour le VIH (2).

Un certain nombre de facteurs peuvent contribuer à la stigmatisation dans les milieux de prestation des services, et la stigmatisation liée aux ITSS, de nature complexe et multidimensionnelle, est un amalgame de tous ces facteurs qui se chevauchent. Ceux-ci comprennent la gêne des fournisseurs de services à discuter de sexualité ou de toxicomanie, les normes sociales stipulant que les personnes touchées par une ITSS sont à blâmer en raison de leur participation à des activités jugées moralement répréhensibles, telles que la débauche sexuelle ou la consommation de drogues, de même que les politiques et procédures organisationnelles qui contribuent par inadvertance à la stigmatisation. Par exemple, dans les milieux de la santé et des services sociaux, les formulaires d'admission n'emploient pas toujours un libellé inclusif, il y a souvent des pénalités pour les rendez-vous manqués et le personnel manque parfois de formation sur les questions liées à la sécurisation culturelle (des pratiques conçues pour que le client ou le patient se sente à l'aise) et à la réduction de la stigmatisation (3,9-13). De plus, la stigmatisation liée aux ITSS ne se produit pas de façon isolée; elle peut aggraver d'autres formes de stigmatisation et d'oppression, comme la stigmatisation associée à la consommation de drogues injectables, à la prostitution, au racisme, au sexisme et à l'homophobie (5), menant ainsi à plusieurs couches de stigmatisation à l'endroit des personnes qui ont plus d'une identité stigmatisée.

Entre avril 2014 et mars 2017, I'Association canadienne de santé publique (ACSP), en partenariat avec de nombreux professionnels et organismes, a créé des ressources d'application des connaissances et des ateliers de formation pour aider les fournisseurs de services de santé et de services sociaux à offrir des services plus sûrs et inclusifs de santé sexuelle, de réduction des méfaits et en matière d'ITSS. Cet article a pour objectif de décrire l'élaboration, le contenu et l'évaluation de ces ressources et ateliers.

\section{Méthodologie}

\section{Activités d'établissement de la portée}

Avant d'élaborer les ressources du projet, I'ACSP a entrepris de nombreuses activités d'établissement de la portée, notamment:

- Une analyse documentaire pour cerner les différentes formes de stigmatisation et les facteurs qui contribuent à la stigmatisation;

- Une analyse du contexte pour relever les ressources d'apprentissage continu auxquelles les fournisseurs de services sociaux et de santé ont accès au Canada;

- Vingt entrevues avec des répondants clés de diverses disciplines, dont l'éducation, la défense des droits, la recherche, la promotion de la santé, la planification des programmes de réduction des méfaits, la sensibilisation communautaire, la médecine, le travail social, le droit, les soins infirmiers et les services pharmaceutiques. Ces répondants clés travaillaient dans des milieux ruraux et urbains d'un bout à l'autre du pays (Ontario : $n=8$, Manitoba : $n=3$, Alberta : $n=2$, Colombie-Britannique $: n=2$, Québec $: n=2$, Nouvelle-Écosse $: n=2$ Nunavut $: n=1$ ) et possédaient divers niveaux d'expertise dans la prestation de services aux personnes touchées de façon disproportionnée par les ITSS;

- Trois groupes de discussion (à Fredericton, au Nouveau-Brunswick [ $n=9$ ], à Toronto, en Ontario [ $n=6$ ] et à Thunder Bay, en Ontario [ $n=12]$ ) avec des personnes touchées par des ITSS afin de recueillir leurs commentaires sur la façon de rendre les milieux des services plus sûrs et plus inclusifs. Les participants aux groupes de discussion ont affirmé vivre une forme intériorisée de stigmatisation en réaction à un diagnostic d'ITSS et ils ont fait écho aux conclusions de la littérature sur les facteurs contribuant à la stigmatisation, comme le jugement moral des fournisseurs de services, notamment au sujet de la diversité des genres, de la diversité sexuelle et de la toxicomanie; la gêne des fournisseurs de services à discuter des activités sexuelles et de la consommation de drogues; le manque de services adaptés aux cultures et aux communautés; le manque de temps pour discuter de questions plus générales ayant une incidence sur la santé, comme le logement et le transport; le coût des traitements; ainsi que la pénurie de services anonymes de dépistage des ITSS et de réduction des effets nocifs dans leur quartier ou leur région.

\section{Élaboration d'un cadre conceptuel}

Les travaux d'établissement de la portée ont mené à l'élaboration d'un cadre conceptuel de la stigmatisation liée aux ITSS, qui a ensuite servi à orienter l'élaboration des ressources du projet (figure 1). Ce cadre met en évidence les diverses formes de stigmatisation relevées dans la littérature : intériorisée, perçue, effective, institutionnelle et composée. Quelques exemples de sources de stigmatisation croisées, comme le racisme, le classisme et l'hétérosexisme, y apparaissent également, de même que les différents niveaux de la société (p. ex., individuel, communautaire, politique/juridique) auxquels la stigmatisation peut se manifester et auxquels on peut la confronter. 
Figure 1 : Définir la stigmatisation liée aux ITSS ${ }^{a}$

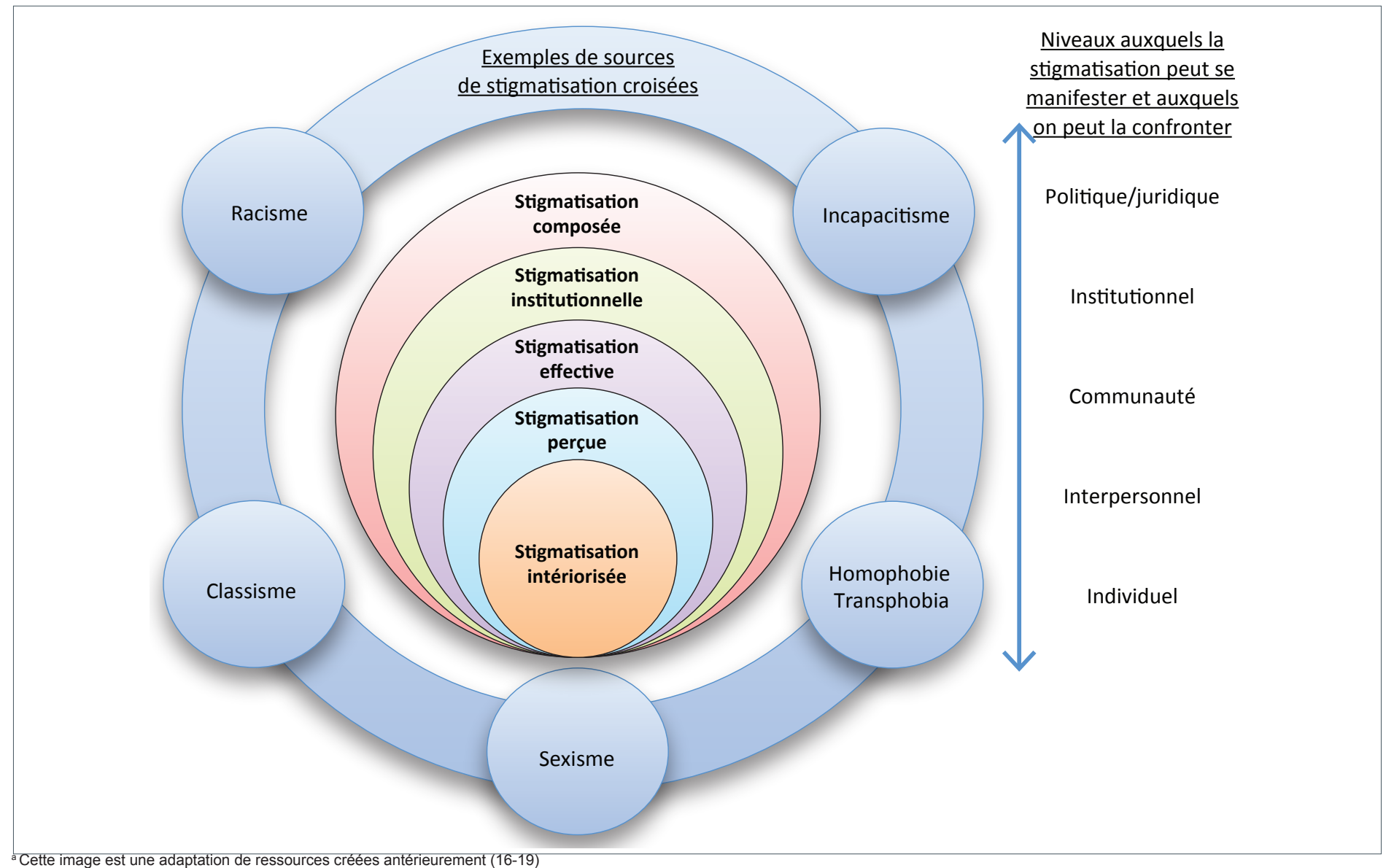

\section{Élaboration des ressources}

Bien que les activités d'établissement de la portée décrites ci-dessus aient permis de relever un certain nombre de ressources axées sur la santé sexuelle et la réduction des effets nocifs, il n'y avait pas suffisamment de ressources canadiennes de formation continue axées sur la réduction de la stigmatisation liée aux ITSS. Pour combler cette lacune, I'ACSP s'est associée à divers experts et organismes communautaires pour élaborer quatre ressources d'application des connaissances : un outil d'autoévaluation permettant aux fournisseurs de services sociaux et de santé de mesurer leurs attitudes et leurs croyances au sujet des ITSS et de la stigmatisation; un guide de discussion visant à favoriser des discussions respectueuses et inclusives sur les questions liées à la sexualité, à la toxicomanie et aux ITSS; une trousse d'outils axée sur la réduction de la stigmatisation, la protection de la vie privée, la confidentialité et la criminalisation de la non-divulgation de la séropositivité; ainsi qu'un outil d'évaluation organisationnelle relatif aux ITSS et à la stigmatisation à l'intention des milieux de la santé et des services sociaux. On a ensuite intégré ces ressources d'application des connaissances à trois ateliers de formation en personne, qui ont été élaborés en partenariat avec le Calgary Sexual Health Centre (CSHC), avant d'être mis à l'essai et évalués pour en assurer la pertinence et l'utilité.

\section{Outil d'autoévaluation}

L'outil d'autoévaluation a été conçu pour aider les fournisseurs de services à réfléchir à leurs attitudes personnelles et à leurs croyances au sujet des ITSS. Cette ressource est une adaptation de l'échelle de stigmatisation du $\mathrm{VIH} /$ sida chez les fournisseurs de soins de santé (14), qui avait déjà été validée antérieurement. On a adapté cette échelle en partenariat avec la $D^{\text {re }}$ Anne Wagner, qui a dirigé l'équipe de recherche ayant conçu l'échelle de stigmatisation originale, afin d'y incorporer un plus grand nombre d'ITSS tout en suivant la tendance qui consiste à adopter une approche plus intégrée pour les aborder (15). L'outil révisé a fait l'objet d'une validation psychométrique après deux séries d'essais pilotes auprès de 144 fournisseurs de services partout au Canada. L'analyse des résultats des essais pilotes a démontré que l'outil d'autoévaluation a une cohérence interne allant de bonne à excellente, ainsi qu'une validité convergente et divergente avec d'autres mesures de la stigmatisation liée au VIH et de la désirabilité sociale. Cependant, cette deuxième constatation doit être interprétée avec prudence en raison de la faible fiabilité des autres mesures de la stigmatisation liée au VIH et de la désirabilité sociale dans cet échantillon.

\section{Guide de discussion à l'intention des fournisseurs de services}

Ce guide de discussion met en évidence les stratégies de communication que les fournisseurs de services peuvent adopter 
pour s'assurer que les discussions sur la sexualité, la toxicomanie et les ITSS sont respectueuses et inclusives. Le guide de discussion est une adaptation du document A guide to taking a sexual health history (20) des Centers for Disease Control and Prevention américains, et il a été révisé à la lumière des pratiques exemplaires relevées dans la littérature; d'entrevues auprès de répondants clés, dont des médecins, du personnel infirmier, du personnel infirmier praticien, des gestionnaires de programmes de santé publique, des pédagogues et des chercheurs; d'essais pilotes menés par des médecins, du personnel infirmier et du personnel infirmier praticien; ainsi que des commentaires formulés par 14 personnes, dont des personnes infectées par le $\mathrm{VIH}$, des consommateurs de drogues et des travailleurs du sexe, ayant participé à trois groupes de discussion tenus à Victoria (Colombie-Britannique), à Winnipeg (Manitoba) et à Toronto (Ontario). À la suite de ces consultations, on a apporté des changements pour garantir que le libellé utilisé dans le guide de discussion était respectueux et inclusif, ainsi que pour y inclure d'autres exemples de conversations.

\section{Réduire la stigmatisation et la discrimination en protégeant la vie privée et la confidentialité}

Élaboré en partenariat avec le Réseau juridique canadien $\mathrm{VIH} /$ sida, ce guide d'orientation a été conçu en réponse aux déclarations des répondants clé, qui ont affirmé que la criminalisation de la non-divulgation de la séropositivité était une source importante de stigmatisation au Canada et un problème dont les fournisseurs de services ont souvent du mal à discuter avec leurs clients et patients. Cette ressource explique le rôle important de la protection de la vie privée et de la confidentialité dans la réduction de la stigmatisation liée aux ITSS. Elle avance plusieurs stratégies que les fournisseurs de services sociaux et de santé peuvent utiliser pour aborder les questions de la protection de la vie privée, de la confidentialité, de la criminalisation de la non-divulgation de la séropositivité et de la réduction de la stigmatisation.

\section{Outil d'évaluation organisationnelle pour les ITSS et la stigmatisation}

Cet outil comprend 30 questions pour aider les organismes de santé et de services sociaux à évaluer leurs forces et leurs faiblesses en matière de prestation de services accueillants et inclusifs dans les domaines de la santé sexuelle, de la réduction des effets nocifs et des ITSS. L'outil d'évaluation a été conçu en s'appuyant sur des interventions éprouvées de réduction de la stigmatisation décrites dans la littérature et après avoir consulté des spécialistes, qui l'ont aussi mis à l'essai.

\section{Ateliers de formation}

On a élaboré trois ateliers de formation fondés sur les principes de l'apprentissage des adultes afin d'aider à accroître la connaissance et l'adoption de stratégies de réduction de la stigmatisation dans les services de santé sexuelle et en matière de réduction des effets nocifs et d'ITSS. Pour assurer la pertinence et l'utilité des ateliers, I'ACSP et le CSHC ont organisé, en 2015 et 2016, 19 ateliers pilotes dans 14 villes canadiennes. Les 589 participants à ces ateliers pilotes comprenaient du personnel infirmier, du personnel infirmier praticien, des travailleurs sociaux, des médecins, des conseillers, des éducateurs en matière de santé, des sages-femmes, etc. Ils possédaient divers niveaux de connaissances et d'expérience en matière de santé sexuelle et de réduction des effets nocifs, mais plusieurs fournissaient des services spécialisés à des groupes de population touchés de façon disproportionnée par les ITSS. Ils ont été invités à remplir des questionnaires d'évaluation avant et après les ateliers pilotes, sauf lors de quelques ateliers où le temps manquait. Les constatations ont servi à réviser la matière de l'atelier et à vérifier sa pertinence compte tenu des besoins $d$ 'apprentissage des fournisseurs de services. Une fois validés sur les plans de la pertinence et de l'utilité, les documents de I'atelier, y compris les manuels des animateurs, les cahiers des participants et les diapositives de présentation, ont été mis à la disposition d'organismes communautaires partout au pays pour soutenir leurs efforts de perfectionnement professionnel.

\section{Évaluation}

La mesure de l'incidence du projet portait principalement sur les changements immédiats dans les niveaux de sensibilisation et de connaissance après la participation aux ateliers pilotes. Parmi les 589 fournisseurs de services sociaux et de santé qui ont participé aux ateliers pilotes, 483 ont été invités à remplir des questionnaires avant et après les ateliers, ce qui s'est traduit par un taux de réponse global de $85 \%$. Les participants ont été invités à noter les connaissances acquises et l'applicabilité de la matière de l'atelier, ainsi qu'à relever les éléments à améliorer dans l'atelier. On a ensuite apporté des changements à la matière en fonction des conclusions de ces évaluations. La grande majorité des participants ont déclaré que les ateliers pilotes leur avaient permis d'acquérir des connaissances, les autres étant surtout des spécialistes. Le tableau 1 présente un résumé de l'analyse des réponses au questionnaire remis après l'atelier, ainsi que des exemples de commentaires formulés par les participants.

Tableau 1 : Résumé de la rétroaction des participants à I'atelier

\begin{tabular}{|c|c|c|}
\hline $\begin{array}{l}\text { Élément évalué } \\
\text { (nombre de } \\
\text { répondants) }\end{array}$ & $\begin{array}{l}\text { Nombre de } \\
\text { répondants en } \\
\text { accord }(\%)\end{array}$ & $\begin{array}{c}\text { Exemples de commentaire } \\
\text { des participants }\end{array}$ \\
\hline $\begin{array}{l}\text { Sensibilisation } \\
\text { accrue aux } \\
\text { diverses formes } \\
\text { de stigmatisation } \\
(\mathrm{n}=397)\end{array}$ & 349 (88 \%) & $\begin{array}{l}\text { "La répartition des différents } \\
\text { types de stigmatisation et de } \\
\text { leur incidence était utile. " }\end{array}$ \\
\hline $\begin{array}{l}\text { Aisance accrue } \\
\text { à discuter de } \\
\text { sexualité, de } \\
\text { consommation } \\
\text { de drogues et } \\
\text { de réduction des } \\
\text { effets nocifs avec } \\
\text { des clients ou des } \\
\text { patients ( } n=378 \text { ) }\end{array}$ & 330 (87 \%) & $\begin{array}{l}\text { "Le jeu de rôle a permis aux } \\
\text { gens de s'exercer à utiliser les } \\
\text { compétences acquises et de } \\
\text { ressentir la gêne que ressent } \\
\text { probablement un usager des } \\
\text { services. " }\end{array}$ \\
\hline $\begin{array}{l}\text { Sensibilisation } \\
\text { accrue aux } \\
\text { stratégies } \\
\text { organisationnelles } \\
\text { visant à réduire } \\
\text { la stigmatisation } \\
(\mathrm{n}=93)\end{array}$ & $84(90 \%)$ & $\begin{array}{l}\text { "Un bon moyen de nous faire } \\
\text { prendre conscience de l'effet } \\
\text { de notre langage, de mieux } \\
\text { faire connaître les différents } \\
\text { types de commentaires que } \\
\text { nous formulons et de réfléchir } \\
\text { à la manière de régler les } \\
\text { problèmes structurels. » }\end{array}$ \\
\hline
\end{tabular}


Tableau 1 : Résumé de la rétroaction des participants à l'atelier (suite)

\begin{tabular}{|l|l|l|}
\hline $\begin{array}{c}\text { Élément évalué } \\
\text { (nombre de } \\
\text { répondants) }\end{array}$ & $\begin{array}{c}\text { Nombre de } \\
\text { répondants en } \\
\text { accord (\%) }\end{array}$ & $\begin{array}{l}\text { Exemples de commentaire } \\
\text { des participants }\end{array}$ \\
\hline $\begin{array}{l}\text { Capacité de } \\
\text { mettre en } \\
\text { pratique ce qui } \\
\text { a été appris en } \\
\text { atelier }(\mathrm{n}=398)\end{array}$ & $372(93 \%)$ & $\begin{array}{l}\text { "J'espère être en mesure } \\
\text { d'apporter des changements } \\
\text { durables au sein de mon } \\
\text { organisation ou, à tout } \\
\text { le moins, de commencer } \\
\text { à avancer dans la bonne } \\
\text { direction. » }\end{array}$ \\
\hline $\begin{array}{l}\text { Commentaires } \\
\text { généraux }\end{array}$ & s/o & $\begin{array}{l}\text { « Cet atelier devrait être } \\
\text { obligatoire pour tous ceux qui } \\
\text { travaillent avec le public, et ce, } \\
\text { à n'importe quel niveau. " }\end{array}$ \\
\hline
\end{tabular}

Abréviation : n, nombre; s/o, sans objet

Les conclusions générales englobent les trois ateliers de formation; étant donné les objectifs d'apprentissage particuliers de chacun, le questionnaire remis après l'atelier était légèrement différent pour chacun, ce qui fait que la taille de l'échantillon pour chaque élément évalué variait également

Les documents de l'atelier ainsi que les ressources complémentaires d'application des connaissances figurent sur le site Web de I'ACSP, à l'adresse https://www.cpha.ca/fr/ les-infections-transmissibles-sexuellement-et-par-le-sang-et-lastigmatisation-associee.

\section{Discussion}

Ce projet a démontré que l'on peut améliorer grandement la capacité des professionnels de la santé et des services sociaux à réduire la stigmatisation lorsqu'ils offrent des services de santé sexuelle, de réduction des effets nocifs et en matière d'ITSS. Grâce aux activités d'établissement de la portée du projet, I'ACSP a pu recueillir les points de vue de personnes touchées par les ITSS et de spécialistes du sujet sur les stratégies optimales pour réduire la stigmatisation dans les milieux de la santé et des services sociaux. Elle a également pu tirer parti de vastes bassins de connaissances et d'expertise en collaborant avec des organisations communautaires et des professionnels durant l'élaboration et la mise à l'essai des ressources. La majorité des participants ont signalé une meilleure connaissance des stratégies de réduction de la stigmatisation liée aux ITSS après avoir participé aux ateliers pilotes. Ils ont également indiqué leur intention de mettre en pratique ce qu'ils ont appris en atelier et de transmettre les ressources de l'atelier à leurs collègues.

Malgré ces succès, on a relevé certaines limites. Le point le plus important est que ce qui a été mesuré après l'atelier portait principalement sur les changements immédiats dans les attitudes et les connaissances. Pour évaluer les changements dans les pratiques des fournisseurs ainsi que les changements dans les politiques et procédures organisationnelles, il faut effectuer un suivi à moyen et à long terme. II convient également de souligner que les ressources du projet ne répondent pas aux besoins particuliers des diverses populations touchées de façon disproportionnée par les ITSS. II pourrait donc être nécessaire d'adapter les ressources au travail avec des groupes de population spécifiques. Enfin, il faut effectuer des efforts supplémentaires pour s'assurer que ces ressources et les autres initiatives similaires atteignent une proportion plus grande de fournisseurs de services canadiens intéressés par les ressources de formation sur la réduction de la stigmatisation liée aux ITSS.

L'ACSP s'est récemment vu attribuer un projet pour continuer à produire des formations à l'intention des fournisseurs de services sociaux et de santé, ainsi que pour travailler avec d'autres organisations afin de soutenir leur utilisation du matériel des ateliers, en particulier lorsque les occasions de formation sont rares. Les travaux futurs mettront aussi l'accent sur l'évaluation des changements à moyen et à long terme (p. ex., au moyen d'enquêtes de suivi après six mois) dans les pratiques des fournisseurs et des organisations.

\section{Conclusion}

La réduction de la stigmatisation liée aux ITSS est toujours un enjeu d'importance pour la santé publique. Ce projet a démontré qu'en intégrant des ressources éprouvées, conviviales et culturellement sécurisantes aux ateliers de formation, on peut améliorer efficacement la capacité des fournisseurs de services à réduire la stigmatisation. Notre évaluation révèle une forte volonté parmi les fournisseurs de services au Canada à profiter de ces occasions.

\section{Déclaration de l'auteur}

R.M. - Conceptualisation, Méthodologie, Administration du projet, Rédaction - ébauche originale, examen et révision

\section{Conflit d'intérêt}

Aucun.

\section{Contributions}

Laura Bouchard, Association canadienne de santé publique Rédaction - examen et révision

\section{Remerciements}

Ce projet a été rendu possible grâce au soutien et à la participation des nombreux organismes et professionnels qui ont examiné les ressources du projet et fourni des commentaires à titre d'experts lors des entrevues avec des répondants clés, des consultations communautaires et des essais pilotes. Nous sommes également redevables aux membres du groupe témoin d'experts du projet qui ont offert des conseils et un soutien spécialisés tout au long des étapes du projet. Enfin, nous aimerions remercier les personnes des diverses communautés qui ont participé aux groupes de discussion et fait part de leurs expériences, de leurs réflexions et de leur sagesse. 


\section{Financement}

Ce projet a été financé par l'Agence de la santé publique du Canada pour la période d'avril 2014 à mars 2017. L'Association canadienne de santé publique a également reçu du financement de l'Agence pour poursuivre ses efforts dans ce domaine d'avril 2017 à mars 2022.

Les opinions exprimées ici ne représentent pas nécessairement les points de vue de l'Agence de la santé publique du Canada.

\section{Références}

1. Le programme commun des Nations Unites sur le $\mathrm{VIH} /$ sida (ONUSIDA). Guide de terminologie de I'ONUSIDA, 2015. Geneva (CH): Le programme commun des Nations Unites sur le VIH/sida ; 2015. http://www.unaids.org/sites/default/files/ media_asset/2015_terminology_guidelines_fr.pdf

2. Rueda S, Mitra S, Chen S, Gogolishvili D, Globerman J, Chambers $L$ et al. Examining the associations between HIV-related stigma and health outcomes in people living with HIV/AIDS: a series of meta-analyses. BMJ Open 2016 Jul;6(7):e011453. DOI (http://dx.doi.org/10.1136/ bmjopen-2016-011453). PubMed (https://www.ncbi.nlm. nih.gov/entrez/query.fcgi?cmd=Retrieve\&db=PubMed\&li st_uids=27412106\&dopt=Abstract).

3. Mill J, Edwards N, Jackson R, Austin W, MacLean L, Reintjes F. Accessing health services while living with HIV: intersections of stigma. Can J Nurs Res 2009 Sep;41(3):168-85. PubMed (https://www.ncbi.nlm.nih. gov/entrez/query.fcgi?cmd=Retrieve $\& d b=$ PubMed\&lis t_uids=19831060\&dopt=Abstract).

4. Foster LR, Byers ES. Predictors of the Sexual Well-being of Individuals Diagnosed with Herpes and Human Papillomavirus. Arch Sex Behav 2016 Feb;45(2):40314. DOI (http://dx.doi.org/10.1007/s10508-0140388-x). PubMed (https://www.ncbi.nlm.nih.gov/ entrez/query.fcgi?cmd=Retrieve \&db=PubMed\&list_ uids $=25408498 \&$ dopt $=$ Abstract).

5. Wagner AC, Girard T, McShane KE, Margolese S, Hart TA. HIV-related stigma and overlapping stigmas towards people living with HIV among health care trainees in Canada. AIDS Educ Prev 2017 Aug;29(4):364-76. DOI (http://dx.doi. org/10.1521/aeap.2017.29.4.364). PubMed (https://www. ncbi.nlm.nih.gov/entrez/query.fcgi?cmd=Retrieve\&db=PubM ed\&list_uids=28825863\&dopt=Abstract).

6. Collins E, Cain R, Bereket T, Chen YY, Cleverly S, George $C$ et al. Living \& serving II: 10 years later - the involvement of people living with HIV/AIDS in the community AIDS movement in Ontario. Toronto (ON): The Ontario HIV Treatment Network; 2007.

7. Worthington C, Jackson R, Mill J, Prentice T, Myers T, Sommerfeldt S. HIV testing experiences of Aboriginal youth in Canada: service implications. AIDS Care 2010 Oct;22(10):1269-76. DOI (https://doi. org/10.1080/09540121003692201). PubMed (https://www. ncbi.nlm.nih.gov/entrez/query.fcgi?cmd=Retrieve \&db=PubM ed\&list_uids=20635240\&dopt=Abstract).
8. Gagnon M. Rethinking HIV-related stigma in health care settings: a research brief. Ottawa (ON): uOttawa; 2014. https://www.cocqsida.com/assets/files/Research-Brief_ RethinkingHIV-17juillet2014.pdf

9. Jaworsky D, Gardner S, Thorne JG, Sharma M, McNaughton N, Paddock S et al.; CHIME Research Group. The role of people living with HIV as patient instructors - reducing stigma and improving interest around HIV care among medical students. AIDS Care 2017 Apr;29(4):524-31. DOI (https:// doi.org/10.1080/09540121.2016.1224314). PubMed (https:// www.ncbi.nlm.nih.gov/entrez/query.fcgi?cmd=Retrieve\&db= PubMed\&list_uids=27577683\&dopt=Abstract).

10. Brener L, Von Hippel W, Kippax S, Preacher KJ. The role of physician and nurse attitudes in the health care of injecting drug users. Subst Use Misuse 2010 Jun;45(7-8):1007-18. DOI (https://doi.org/10.3109/10826081003659543).

PubMed (https://www.ncbi.nlm.nih.gov/entrez/ query.fcgi?cmd=Retrieve\&db=PubMed\&list_ uids $=20441447 \&$ dopt $=$ Abstract).

11. Fuzzell L, Fedesco HN, Alexander SC, Fortenberry JD, Shields CG. "I just think that doctors need to ask more questions": sexual minority and majority adolescents' experiences talking about sexuality with healthcare providers. Patient Educ Couns 2016 Sep;99(9):1467-72. DOI (https:// doi.org/10.1016/j.pec.2016.06.004). PubMed (https://www. ncbi.nlm.nih.gov/entrez/query.fcgi?cmd=Retrieve\&db=PubM ed\&list_uids=27345252\&dopt=Abstract).

12. Gott M, Galena E, Hinchliff S, Elford H. "Opening a can of worms": GP and practice nurse barriers to talking about sexual health in primary care. Fam Pract 2004 Oct;21(5):528-36. DOI (https://doi.org/10.1093/ fampra/cmh509). PubMed (https://www.ncbi.nlm.nih. gov/entrez/query.fcgi?cmd=Retrieve \&db=PubMed\&lis t_uids=15367475\&dopt=Abstract).

13. Nyblade L, Stangl A, Weiss E, Ashburn K. Combating HIV stigma in health care settings: what works? J Int AIDS Soc 2009 Aug;12(1):15. DOI (https://doi. org/10.1186/1758-2652-12-15). PubMed (https://www.ncbi. $\mathrm{nlm}$.nih.gov/entrez/query.fcgi?cmd=Retrieve $\& \mathrm{db}=$ PubMed\&l ist_uids=19660113\&dopt=Abstract).

14. Wagner AC, Hart TA, McShane KE, Margolese S, Girard TA. Health care provider attitudes and beliefs about people living with HIV: Initial validation of the Health Care Provider HIV/AIDS Stigma Scale (HPASS). AIDS Behav 2014 Dec;18(12):2397-408. DOI (https://doi.org/10.1007/ s10461-014-0834-8). PubMed (https://www.ncbi.nlm.nih. gov/entrez/query.fcgi?cmd=Retrieve $\& \mathrm{db}=$ PubMed\&lis t_uids=24965675\&dopt=Abstract).

15. Paquette D. Integrated approach to the prevention and control of sexually transmitted and blood borne infections. Ottawa (ON): Public Health Agency of Canada. http://www. catie.ca/sites/default/files/2-845-1-DanaPaquette.pdf

16. Churcher S. Stigma related to HIV and AIDS as a barrier to accessing health care in Thailand: a review of recent literature. WHO South East Asia J Public Health 2013 Jan-Mar;2(1):12-22. DOI (http://dx.doi. org/10.4103/2224-3151.115829). PubMed (https://www.ncbi. 
nlm.nih.gov/entrez/query.fcgi?cmd=Retrieve\&db=PubMed\&l ist_uids=28612818\&dopt=Abstract).

17. Loutfy MR, Logie $\mathrm{CH}$, Zhang Y, Blitz SL, Margolese SL, Tharao WE et al. Gender and ethnicity differences in HIV-related stigma experienced by people living with HIV in Ontario, Canada. PLoS One 2012;7(12):e48168. DOI (http://dx.doi. org/10.1371/journal.pone.0048168). PubMed (https://www. ncbi.nlm.nih.gov/entrez/query.fcgi?cmd=Retrieve\&db=PubM ed\&list_uids=23300514\&dopt=Abstract).

18. Stangl AL, Brady L, Fritz K. Measuring HIV stigma and discrimination: Technical Brief July 2012. Washington, DC: International Centre for Research on Women; 2012. http://strive.Ishtm.ac.uk/system/files/attachments/STRIVE_ stigma\%20brief-A4.pdf
19. Corrigan PW, Markowitz FE, Watson AC. Structural levels of mental illness stigma and discrimination. Schizophr Bull 2004;30(3):481-91. DOI (http://dx.doi.org/10.1093/ oxfordjournals.schbul.a007096). PubMed (https://www.ncbi. $\mathrm{nlm}$.nih.gov/entrez/query.fcgi?cmd=Retrieve\&db=PubMed\&l ist_uids=15631241\&dopt=Abstract).

20. A guide to taking a sexual health history. Atlanta (GA): Centers for Disease Control and Prevention, US National Center for HIV Viral Hepatitis STD and TB Prevention; 2011. http://www.cdc.gov/std/treatment/SexualHistory. pdfJan-Mar;2(1):12-22. http://dx.doi.org/10.4103/22243151.115829

\section{Lignes directrices canadiennes sur les infections transmissibles sexuellement}

\section{Mise à jour de l'application mobile (mai 2017)}
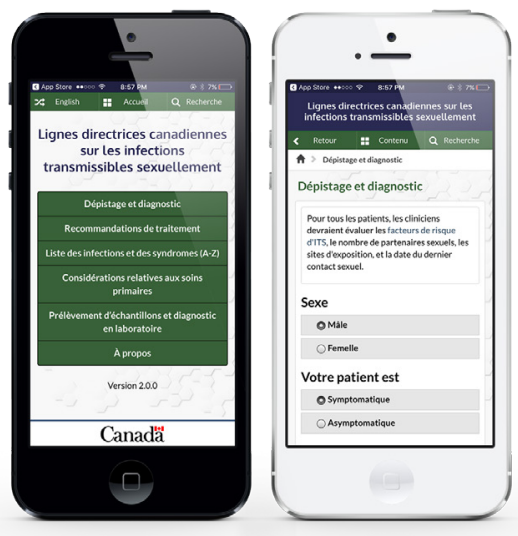

Agence de la santé Public Health

publique du Canada Agency of Canada
Nouveautés de la version 2.0.0

- Amélioration de l'interactivité avec des hyperliens internes

- Des boîtes contextuelles avec des renseignements supplémentaires et de conseils

- Des hyperliens externes vers des ressources complémentaires

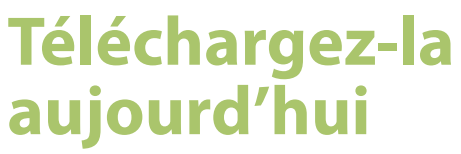

Téléchargement gratuit C. Avaitbole on the $D$ Google Play

Recherchez:

«Lignes directrices CDN ITS » 